\title{
La mise en place du Réseau national du savoir
}

Préparer le Royaume-Uni à l'ère de l'information

\section{Rachid R'Kaina}

\section{CpenEdition}

Journals

Édition électronique

URL : http://journals.openedition.org/ries/2896

DOI : 10.4000/ries.2896

ISSN : 2261-4265

Éditeur

Centre international d'études pédagogiques

Édition imprimée

Date de publication : 1 juin 1998

Pagination : 37-44

ISSN : $1254-4590$

Référence électronique

Rachid R'Kaina, "La mise en place du Réseau national du savoir », Revue internationale d'éducation de Sèvres [En ligne], 18 | juin 1998, mis en ligne le 18 avril 2013, consulté le 01 mai 2019. URL : http:// journals.openedition.org/ries/2896 ; DOI : 10.4000/ries.2896

Ce document a été généré automatiquement le 1 mai 2019.

(c) Tous droits réservés 


\title{
La mise en place du Réseau national du savoir
}

\author{
Préparer le Royaume-Uni à l'ère de l'information
}

\author{
Rachid R'Kaina
}

\section{NOTE DE L'AUTEUR}

Cet article a été élaboré à partir du document Connecting the Learning Society, National Grid for Learning, The Government's Consultation Paper et de documents émanant du Department for Education and Employment, du Education Departments' Superhighways Initiative, de l'Office for Standards in Education et de la Qualifications and Curriculum Agency.

1 En octobre 1997, avec la publication du rapport gouvernemental Connecting the Learning Society, la possibilité d'utiliser un ensemble de réseaux électroniques interconnectés pour relier les enseignants et les apprenants de tous âges aux ressources idoines a dépassé le stade de la promesse électorale.

2 L'an dernier, le Premier ministre Tony Blair a demandé à des personnalités indépendantes une enquête sur le potentiel de la télématique dans le système scolaire. Les conclusions de cette enquête ont permis de cerner deux problèmes : la nécessité de former les enseignants et celle de créer un marché pour les logiciels éducatifs de qualité.

3 Le financement de la formation des enseignants à l'informatique, qui doit être pris en charge par la Loterie nationale, comme l'annonce un livre blanc publié récemment, réglera le premier problème. Le Réseau national constituera l'outil grâce auquel le second problème sera pris en charge. 


\section{La société apprenante à l'ère de l'information} l'enseignement et la formation permanente des progrès des technologies de communication en réseaux et de permettre la concrétisation, grâce à la création du Réseau national du savoir, de notre conception d'une société apprenante.

Pour la première fois, nous est offerte la possibilité de connecter entre eux tous les organismes d'enseignement et de formation - aussi bien les écoles, les établissements postscolaires, les universités, les bibliothèques que les centres de formation pour adultes, les musées et les galeries d'art - et, qui plus est, de les relier dans le but de mettre en œuvre un programme qui permettra de construire la société apprenante. Pour rendre cette société possible, il faut que les liens mis en place se prolongent de façon efficace jusqu'à chaque foyer, jusqu'aux lieux de travail, aux hôpitaux, aux centres villes et au moindre coin de rue, de la même façon que des services publics tels que le téléphone sont aujourd'hui universellement accessibles.

6 Il y a un certain nombre d'années que l'on prédit la convergence des technologies du téléphone, de la télévision et de l'informatique. Cette convergence a lieu en ce moment même : Internet a déjà réalisé la fusion de l'informatique et des télécommunications, et la télévision numérique interactive doit être lancée l'an prochain en Grande-Bretagne. On peut à présent utiliser le numérique pour transmettre un volume d'informations considérable (qu'il s'agisse de texte, d'images fixes, de sons ou de vidéo) en empruntant toute une gamme de moyens de communication - du réseau téléphonique classique aux réseaux de fibre optique, des ondes hertziennes à la transmission par satellite.

7 Plus la technologie progresse, plus le débit des transmissions s'accélère. La connexion à un réseau, y compris à Internet (le réseau mondial des réseaux), est d'ores et déjà possible depuis un ordinateur et elle sera bientôt réalisable par le biais de la télévision. Les réseaux à haut débit (les autoroutes de l'information) sont de plus en plus nombreux et, à mesure que leur capacité augmente, nous assistons à un accroissement spectaculaire de la richesse et de la diversité des contenus qu'ils véhiculent. Ceci représente un potentiel énorme, aussi bien pour l'éducation que pour le monde des affaires ou d'autres secteurs de la société.

Le Livre blanc L'excellence à l'école, récemment publié en Angleterre, et son équivalent gallois s'appuient sur l'engagement du gouvernement, diffusé sous forme de manifeste à l'échelle du Royaume-Uni, d'incorporer la télématique à l'enseignement. Ces documents présentent des propositions destinées à moderniser l'enseignement du siècle prochain, en intégrant, par exemple, le déploiement des technologies nouvelles à un programme destiné à rehausser les niveaux moyens d'éducation et qui concernerait aussi bien les nouveaux seuils de maitrise de l'écriture et du calcul, la formation professionnelle des enseignants, les écoles pilotes, une nouvelle base de données nationale des pratiques professionnelles, les activités d'apprentissage hors emploi du temps scolaire et les programmes pour les enfants ayant des besoins spécifiques.

9 La réalisation de ces propositions ne sera possible que par la mise en place du nouveau Réseau national du savoir, à la fois infrastructure et service d'accès aux sources de connaissances. Le Réseau ne sera pas un service proposé par une seule compagnie, mais sera une architecture rassemblant une mosaïque de réseaux et de services interconnectés. 
Le projet est de consacrer le Réseau, dans un premier temps, à la formation des enseignants et au secteur scolaire, avant de l'étendre à la formation permanente - qu'il s'agisse de formation personnelle à domicile dans les domaines des études postscolaires ou universitaires, ou de recyclage professionnel en vue d'une recherche d'emploi. Il fonctionnera en relation étroite avec le plan de formation des enseignants aux techniques de la télématique, financé par la Loterie nationale, et les projets concernant l'université pour l'industrie. Les musées nationaux et locaux, les galeries d'art et autres sources de contenus culturels auront un rôle important à jouer. Il est prévu d'intégrer totalement au Réseau les bibliothèques et leurs stocks d'informations considérables facilement accessibles au public. De cette façon, le Réseau rendra disponibles pour tous les apprenants les richesses de l'héritage intellectuel, culturel et scientifique du monde entier. Comme l'information peut être distribuée sur Internet de façon pratiquement gratuite, le Réseau ouvrira à tous les portes du savoir qui ne sera plus confiné à la stricte enceinte des établissements d'enseignement.

Une partie essentielle du programme de mise en place du Réseau consistera à développer les compétences des enseignants et des bibliothécaires. Cette étape pourra être assurée grâce au financement de la Loterie nationale. Des propositions en ce sens figurent sous forme détaillée dans le document sur la consultation concernant la "Loterie populaire ». Nous envisageons une action complémentaire à celle exposée dans ce document, qui assurera aux enseignants la meilleure formation possible à une utilisation particulière de la télématique en rapport direct avec toutes les matières enseignées. Il s'agira de dépasser le stade de la simple initiation aux technologies des réseaux informatiques, qui se contente d'une sensibilisation à l'informatique et à l'utilisation de logiciels tels que les traitements de texte, pour aller vers la découverte d'applications capables d'aider les professeurs à découvrir de nouvelles façons d'utiliser efficacement la télématique dans leurs classes pour enseigner leur matière. Ces nouvelles approches pourraient inclure la possibilité d'offrir une pédagogie différenciée aux enfants qui ont des besoins spécifiques. À terme, cette formation pourrait être dispensée par le biais du Réseau lui-même. Des dispositions semblables seront prises pour la formation des bibliothécaires.

\section{La tâche à accomplir}

11 Il ne faut pas sous-estimer l'ampleur du travail que représente la création d'un réseau du savoir à une échelle qui soit véritablement nationale. Au Royaume-Uni, il existe 32000 écoles (qu'elles soient indépendantes ou financées par l'État), ce qui représente 450000 enseignants et plus de 9 millions d'élèves. Il y a 540 établissements d'enseignement postscolaire et 360 autres institutions s'adressant au même public, qui emploient ensemble quelque 250000 personnes et accueillent environ 4 millions d'étudiants. L'enseignement supérieur emploie environ 380000 personnes et concerne plus de 16 millions d'étudiants. Il existe 4300 bibliothèques, où travaillent 22000 personnes, dont plus de 6000 bibliothécaires de formation, et que fréquentent chaque année plus de $60 \%$ de la population adulte. De plus, il y a des milliers d'institutions et d'organismes publics et privés, parmi lesquels les musées, les galeries d'art, les chaînes de radio et de télévision, les autorités locales, les centres d'emploi et de formation, les comités d'initiative locale en Écosse, les organismes de formation professionnelle de l'industrie, les organismes nationaux de formation, les associations caritatives et les groupes d'aide aux handicapés, dont la présence pourrait enrichir le Réseau. 

que pour être véritablement efficace, le Réseau national du savoir doit apporter son soutien à la fois à l'enseignement scolaire et à la formation permanente sur des bases aussi larges que possible. Il doit aussi constituer une aide pour un vaste éventail d'initiatives dans le domaine de l'éducation, de la formation permanente et de l'aide sociale, telles que l'Université pour l'industrie et le programme «Du chômage à l'emploi ». Ses contenus doivent être exhaustifs, pertinents, diversifiés en fonction des besoins, et facilement accessibles.

Les actions spécifiques proposées et décrites en détail dans ce document consistent à :

- mener une large consultation sur les principes et les mécanismes qui permettront de créer un Réseau national du savoir ;

- assigner à l'emploi de la télématique dans l'éducation des objectifs ambitieux qui devront aussi permettre d'atteindre un haut niveau pour les enjeux nationaux en matière de maîtrise de l'écriture et du calcul, afin que le Réseau, en tant que ressource, soit utilisé au mieux ;

- installer un prototype du Réseau répondant initialement aux besoins des enseignants et des écoles pour mettre ces idées à l'épreuve de la pratique et pour stimuler le développement de contenus de grande qualité.

\section{Les besoins et le potentiel}

\section{Tirer le meilleur parti de ce qui est disponible}

Le nombre et la diversité des gens susceptibles d'utiliser le Réseau et d'y apporter leur contribution devrait cependant constituer un atout. Il faudra aussi que le gouvernement, pour sa part, prenne l'initiative de définir la marche à suivre, de fournir un cadre de référence et d'offrir diverses incitations, financières et autres, pour garantir la qualité. Tous les acteurs concernés devront s'engager à travailler de concert, dans un esprit de création collective. Les écoles et le ministère de l'éducation ont une longue tradition de travail en commun lorsqu'il s'agit de transformer en avantage d'apparentes faiblesses, par exemple, en opérant des regroupements, dans des ensembles d'écoles isolées, pour pallier certaines lacunes dans les compétences nécessaires à l'application des programmes officiels, ou encore en suscitant des partenariats dans les quartiers défavorisés. De même, la coopération entre diverses bibliothèques, lorsqu'il s'agit d'obtenir des livres ou d'autres documents pour un public varié et exigeant, est solidement établie.

\section{La compétence et l'assurance des enseignants}

15 Il est d'une importance primordiale de faire progresser les compétences et la confiance en soi des enseignants qui utilisent la télématique ; cela est à présent largement reconnu, et c'est ce que reflètent les conclusions d'une réunion récente des ministres de l'Éducation de l'Union européenne. Cependant, une étude menée en 1996 par le ministère britannique de l'Éducation et de l'Emploi (DfEE) révèle une baisse, aussi bien dans le primaire que dans le secondaire, du pourcentage d'enseignants qui se sentent parfaitement capables d'utiliser la télématique dans leurs classes. Dans le secondaire, les enseignants qui maîtrisent le mieux ces technologies sont ceux dont la matière est traditionnellement liée à l'informatique, comme l'économie et les mathématiques. En revanche, 60 \% seulement 
des professeurs d'anglais, d'histoire, de géographie, de langues étrangères et de sciences se déclarent prêts à utiliser l'informatique.

L'an dernier, pour la première fois, les écoles primaires ont connu une baisse du pourcentage des enseignants ayant bénéficié de plus d'un stage court de formation continue (stage de perfectionnement en télématique ou d'application de la télématique à l'enseignement $\mathrm{du}$ programme). La proportion d'enseignants n'ayant reçu aucune formation dans ce domaine était en augmentation. L'enseignement secondaire n'était pas logé à meilleure enseigne, la proportion des enseignants sans formation stagnant à $14 \%$. Un résultat encourageant: les chiffres révélaient que les enseignants ayant suivi des stages de télématique appliquée à leur matière étaient aussi ceux qui se sentaient le plus à même d'utiliser ces technologies dans leurs classes, ce qui confirme l'intérêt que présente une formation en profondeur après une première étape d'initiation.

\section{Repérer les meilleures expériences pratiques pour s'en inspirer}

17 De nouvelles pistes indiquant les meilleures façons d'aborder l'enseignement et l'apprentissage assistés par la technologie des réseaux seront bientôt disponibles, grâce à la publication séparée de l'évaluation des projets pilotes lancés par le «Plan pour les autoroutes de l'information » du ministère de l'Éducation. À travers le Royaume-Uni, un millier d'écoles et trente établissements d'enseignement postscolaire y ont pris part. Les projets, très variés, ont consisté par exemple à :

- équiper des enseignants (dont certains préparant le concours national de chef d'établissement) d'ordinateurs portables multimédia connectables à Internet ;

- monter des réseaux complets, grâce à l'équipement en ordinateurs d'un nombre substantiel d'écoles et la fourniture d'une grande variété de supports pédagogiques ;

- inciter des écoles spécialisées dans l'utilisation de pointe de la technologie des réseaux à mettre à profit leur compétence pour améliorer la façon d'enseigner certains points des programmes scolaires;

- mettre en pratique quelques expériences de liaison par réseau entre l'école et le domicile des élèves.

\section{La dimension européenne}

18 La Commission européenne donne la priorité à la télématique en soutenant des projets qui affichent clairement une dimension européenne. La Commission européenne prévoit de travailler conjointement avec les États membres, pour promouvoir la connexion de toutes les écoles de l'Union européenne à des réseaux d'information et de savoir à mesure que l'on avancera dans l'ère de l'information.

Grâce à la publication de son plan d'action récent « Les apprentissages dans la société de l'information », la Commission réunira les initiatives locales, régionales et nationales et contribuera à donner une dimension européenne à l'enseignement par le biais des communications électroniques, stimulant le développement d'un marché européen pour le multimédia éducatif. Afin de guider le développement des États membres, il serait possible de rendre accessibles sur Internet les recherches financées par la Commission.

Les organismes d'enseignement du Royaume-Uni sont déjà engagés dans un certain nombre de projets pilotes, par exemple, au sein des programmes européens SOCRATES et «La télématique pour l'enseignement et la formation ». Dans le cadre d'un programme 
des pays du G7, le Royaume-Uni a joué un rôle significatif dans le développement du projet Lingu@NET, dont l'objectif est de créer des centres virtuels d'enseignement et d'apprentissage des langues. Le Royaume-Uni sera un acteur majeur dans le projet, plus large encore, du Réseau des écoles européennes, qui reliera les réseaux scolaires nationaux.

\section{Concevoir le Réseau}

C'est en fonction de ce contexte préexistant qu'il faut concevoir et développer le Réseau national du savoir. Ce Réseau devra se révéler utile. Il faudra qu'il permette aux enseignants, aux bibliothécaires et à d'autres intermédiaires clés d'améliorer leurs compétences et de prendre de l'assurance ; il devra être capable de motiver les élèves et les autres apprenants. D'ores et déjà, il est clair que le Réseau devra constituer :

- un fonds de ressources valable pour les enseignants, les bibliothécaires et les formateurs dans leur travail quotidien et, pour leur propre formation permanente en télématique, par exemple grâce à des didacticiels consultables à distance ou à des aides en ligne ;

- un fonds de ressources capable d'aider les élèves et les autres apprenants à améliorer leurs compétences, particulièrement dans la maîtrise de l'écriture et du calcul ;

- un service qui offre à tout moment un accès aux compétences des experts et à toutes sortes de ressources;

- une structure qui stimule le développement, la diffusion, et l'utilisation de contenus éducatifs de qualité, parmi lesquels des logiciels, des services en lignes et à terme, peut-être, des programmes basés sur la télévision numérique interactive ;

- l'égalité d'accès pour tous les apprenants, qu'ils soient à l'école, à l'université, chez eux ou dans des bibliothèques; qu'ils résident dans des zones rurales ou urbaines ; qu'ils aient un emploi ou qu'ils en cherchent un; que l'anglais soit ou non leur langue maternelle - sans oublier ceux qui souffrent d'un handicap ;

- un moyen d'améliorer et d'harmoniser la gestion des établissements d'enseignement scolaire ou postscolaire, des bibliothèques et autres institutions, en conjonction avec les projets menés par les instances locales et nationales; une structure permettant d'équiper de réseaux internes et de connexions sur l'extérieur: les écoles, les établissements d'enseignement postscolaire, les organismes de formation permanente, en y associant les centres d'éducation hors site et les organisations de jeunesse ;

- l'inter connectivité entre les réseaux et un moyen d'assurer l'utilisation la plus efficace possible des réseaux et des équipements existants;

- une incitation à l'investissement et à la participation pour un vaste éventail d'entreprises industrielles;

- un lien entre de nombreux aspects divers de l'activité humaine, dont le commerce, la culture, le sport, l'enseignement et l'éducation permanente, la santé, les actions caritatives et la politique ;

- un accès abordable, grâce à une baisse des coûts des connexions, de l'équipement et de l'utilisation;

- une procédure de renouvellement de ses services, de ses équipements et de ses systèmes à mesure que la technologie progresse. 


\section{Des objectifs pour la télématique}

La création d'un centre national de ressources d'une telle envergure nécessitera beaucoup de dévouement, d'efforts et de travail en collaboration. Des objectifs ambitieux sont proposés à l'utilisation de la télématique dans l'enseignement et la formation permanente, afin de garantir que le Réseau, en tant que centre de ressources, ne sera pas inutile. Ces objectifs se définissent ainsi :

- dès 1998, devrait commencer le processus de mise en œuvre des structures du Réseau, telles qu'elles auront été définies par les résultats de la présente consultation ;

- en 1999, tous les enseignants nouvellement formés devront faire preuve d'un niveau obligatoire de maîtrise de la télématique pour recevoir leur validation finale (nous avons déjà demandé aux centres de formation pédagogique de définir des critères d'exigence plus spécifiques à l'usage des réseaux afin de les inclure au programme national d'initiation à l'informatique);

- en 2002, les enseignants en exercice devraient posséder, d'une façon générale, la compétence et l'assurance nécessaires à l'utilisation de la télématique dans le cadre des matières inscrites au programme. Grâce à des initiatives telles que le financement par la Loterie populaire des formations en télématique et l'introduction de la télématique au concours national de chef d'établissement, cette maîtrise de la technologie des réseaux devrait être valorisée, considérée comme très importante pour l'enseignement des programmes scolaires et susceptible de permettre une amélioration de carrière (actuellement, selon les chiffres communiqués par les écoles, $70 \%$ des enseignants du primaire et $60 \%$ de ceux du secondaire se sentent en mesure d'utiliser la télématique) ;

- en 2002, toutes les écoles, les établissements postscolaires, les universités, les bibliothèques et le plus grand nombre possible de centres socioculturels devraient être connectés au Réseau, permettant à peut-être $75 \%$ des enseignants et des assistants et à $50 \%$ des élèves d'utiliser leur propre adresse e-mail (actuellement, 20 à $25 \%$ des écoles, par exemple, sont reliées à Internet et, parmi elles, il n'y en a que quelques-unes dont tous les enseignants et tous les élèves possèdent leur propre adresse électronique) ;

- en 2002, la plupart des élèves quittant le système scolaire devraient avoir une bonne connaissance de la télématique, solidement fondée sur les principes décrits dans les programmes appliqués dans diverses parties du Royaume- Uni et des mesures devraient être mises en place pour évaluer leur degré de maîtrise (actuellement, peu nombreux sont ceux qui sortent du système avec une qualification spécifique en télématique).

\section{RÉSUMÉS}

La mise en place du Réseau national du savoir, au Royaume-Uni, est un programme ambitieux qui doit répondre aux attentes de la société apprenante, telle qu'elle est définie par la Commission européenne. Définition des besoins, moyens mis en œuvre et objectifs font l'objet d'une présentation exhaustive. 
INDEX

Mots-clés : formation des enseignants, formation professionnelle, nouvelles technologies, réseau d'information, réseau informatique

Index géographique : Grande-Bretagne, Royaume-Uni

\section{AUTEUR}

\section{RACHID R'KAINA}

TEP Co-ordinator External Relation and Marketing, Technology Enhancement Programme, Londres, Grande-Bretagne 